\begin{tabular}{c} 
Global Journal of Mathematical Analysis, $5(1)(2017) 16-18$ \\
Global Journal of Mathematical Analysis \\
SPC \\
Website: $\begin{array}{c}\text { www.sciencepubco.com/index.php/GJMA } \\
\text { doi: } 10.14419 / \text { gima.v5il.7074 } \\
\text { Short communication }\end{array}$ \\
\hline
\end{tabular}

\title{
The soliton solution of a modified nonlinear schrödinger equation
}

\author{
Jumei Zhang ${ }^{1}$ and Li Yin ${ }^{2}$ * \\ ${ }^{1}$ Department of Mathematics, Binzhou University, Binzhou City, Shandong Province, 256603, China \\ ${ }^{2}$ Department of Mathematics, Binzhou University, Binzhou City, Shandong Province, 256603, China \\ ${ }^{*}$ Corresponding author E-mail: yinli7979@163.com
}

\begin{abstract}
Hirota bilinear derivative method can be used to construct the soliton solutions for nonlinear equations. In this paper we construct the soliton solutions of a modified nonlinear Schrödinger equation by bilinear derivative method.
\end{abstract}

Keywords: nonlinear Schrödinger equation; soliton solution; bilinear derivative.

\section{Introduction}

The study of the exact solutions of nonlinear equations plays an important role in the research of nonlinear physical phenomena. The exact solution can facilitate the verification of numerical solvers and aids in the stability of solutions. In the past years, there has been vital progression in the development of these methods such as algebra-geometric method, Darboux transformation [3, 4], inverse scattering method [1, 2], Hirota bilinear method [5, 6] and so on.

As is well known, the Hirota bilinear derivative method is a powerful and direct method to construct exact solutions of nonlinear equations. Once a nonlinear equation is written in bilinear forms by a dependent variable transformation, then multi-soliton solutions and rational solutions can be attained easily. In this paper, we use Hirota bilinear method to construct the soliton solution of Schrödinger equation

$\mathbf{i} u_{t}+u_{x x}+\mathbf{i} \lambda\left[u^{2} u^{*}\right]_{x}=0$

where $u=u(x, t)$ is a complex-valued function of $x$ and $t$, the asterisk appended to $u$ denotes complex conjugate of $u, \lambda$ is real constant and subscripts $x$ and $t$ appended to $u$ denote partial differentiations.

\section{Preliminary knowledge}

Suppose $f(t, x)$ and $g(t, x)$ are derivative complex-valued functions of $t$ and $x$. The Hirota bilinear operators $D_{t}$ and $D_{x}$ are defined by,

$D_{t}^{m} D_{x}^{n}(f \cdot g)=\left.\left(\partial_{t}-\partial_{t^{\prime}}\right)^{m}\left(\partial_{x}-\partial_{x^{\prime}}\right)^{n} f(t, x) g\left(t^{\prime}, x^{\prime}\right)\right|_{t^{\prime}=t, x^{\prime}=x}$

where $m$ and $n$ are nonnegative integers [5].

In particular,

If $m=0, n=1$, then $D_{t}(f \cdot g)=f_{t} g-f g_{t}$.

If $m=n=1$, then $D_{x} D_{t}(f \cdot g)=f_{x t} g-f_{x} g_{t}-f_{t} g_{x}+f g_{x t}$.

\section{The soliton solutions of the modified nonlin- ear schrödinger equation}

Firstly, substituting the transformations

$u=q \exp \left(-\frac{\mathbf{i} \lambda}{2} \int_{-\infty}^{x} q q^{*} d x\right)$,

into the system (1), we get the system

$\mathbf{i} q_{t}+q_{x x}+\mathbf{i} \lambda q q^{*} q_{x}=0$,

where the asterisk denotes complex conjugate. By the dependent variable transformations

$q=\frac{g}{f}$,

the system (4) can be transformed to the following bilinear forms

$\left(\mathbf{i} D_{t}+D_{x}^{2}\right) g \cdot f=0$,

$D_{x} f \cdot f^{*}=\frac{\mathbf{i} \lambda}{2} g g^{*}$,

$D_{x}^{2} f \cdot f^{*}=\frac{\mathbf{i} \lambda}{2} D_{x} g \cdot g^{*}$.

It follows from (5) and (7) that

$-\frac{\mathbf{i} \lambda}{2} q q^{*}=\frac{\partial}{\partial x} \ln \frac{f^{*}}{f}$

Substituting (8) into (4), we can claim that the soliton solutions of the system (1) in the form

$$
u=\frac{g f^{*}}{f^{2}} .
$$


Suppose $f$ and $g$ can be expanded as the following

$f=1+f^{2} \varepsilon^{2}+f^{4} \varepsilon^{4}+f^{6} \varepsilon^{6}+\cdots+f^{(2 j)} \varepsilon^{2 j}+\cdots$,

$g=g^{1} \varepsilon^{1}+g^{3} \varepsilon^{3}+g^{5} \varepsilon^{5}+\cdots+g^{(2 j+1)} \varepsilon^{2 j+1}+\cdots$.

Substituting (9) and (10) into (6) and (7), then we compare with the same power coefficients of $\varepsilon$ in (7), we find that (7) can be decoupled to a set of equations

$\mathbf{i} g_{t}^{1}+g_{x x}^{1}=0$

$\mathbf{i} g_{t}^{3}+g_{x x}^{3}=-\left(\mathbf{i} D_{t}+D_{x}^{2}\right) g^{1} \cdot f^{2}$,

$\mathbf{i} g_{t}^{5}+g_{x x}^{5}=-\left(\mathbf{i} D_{t}+D_{x}^{2}\right)\left(g^{1} \cdot f^{4}+g^{3} \cdot f^{2}\right)$,

$\mathbf{i} g_{t}^{7}+g_{x x}^{7}=-\left(\mathbf{i} D_{t}+D_{x}^{2}\right)\left(g^{1} \cdot f^{6}+g^{3} \cdot f^{4}+g^{5} \cdot f^{2}\right)$

$f_{x}^{2}-f_{x}^{2 *}=\frac{\mathbf{i} \lambda}{2} g^{1} g^{1 *}$

$f_{x}^{4}-f_{x}^{4 *}=-D_{x} f^{2} \cdot f^{2 *}+\frac{\mathbf{i} \lambda}{2}\left(g^{1} g^{3 *}+g^{3} g^{1 *}\right)$,

$f_{x}^{6}-f_{x}^{6 *}=$

$-D_{x} f^{2} \cdot f^{4 *}-D_{x} f^{4} \cdot f^{2 *}+\frac{\mathbf{i} \lambda}{2}\left(g^{1} g^{5 *}+g^{3} g^{3 *}+g^{1 *} g^{5}\right)$,

$f_{x}^{8}-f_{x}^{8 *}=-D_{x} f^{2} \cdot f^{6 *}-D_{x} f^{4} \cdot f^{4 *}$

$-D_{x} f^{6} \cdot f^{2 *}+\frac{\mathbf{i} \lambda}{2}\left(g^{1} g^{7 *}+g^{3} g^{5 *}+g^{5 *} g^{3}+g^{7 *} g^{1}\right)$,

We can assume that

$g^{1}=e^{\tau_{1}}, \tau_{1}=\alpha_{1} t+\beta_{1} x+\eta_{1}^{0}, \beta_{1}^{2}=-\mathbf{i} \alpha_{1}$

is a solution of homogeneous equation (11), where $\alpha_{1}$ and $\eta_{1}^{0}$ are complex-valued constants. By solving equation (15), we can get

$f^{2}=e^{\tau_{1}+\tau_{1}^{*}+\vartheta_{13}}, e^{\vartheta_{13}}=\frac{\lambda}{2\left(\alpha_{1}^{2}-\alpha_{1}^{* 2}\right)}$,

Substituting $g^{1}$ and $f^{2}$ into (12), then (12) can be written to the former homogeneous equation (11), namely $g_{x x}^{3}+\mathbf{i} g_{t}^{3}=0$. Hence $g^{3}=0$ is a solution of $g_{x x}^{3}+\mathbf{i} g_{t}^{3}=0$, by virtue of $(16)$, then we can get $f^{4}=0$.

The above procedure can be used to (11) - (18) again, then we can get $g^{5}=f^{6}=\cdots=0$. Then we can infer, if $\varepsilon=1$, the series (9) and (10) can be written to

$f_{1}(t, x)=1+e^{\tau_{1}+\tau_{1}^{*}+\vartheta_{13}}, g_{1}(t, x)=e^{\tau_{1}}$.
So, the single soliton solution of system (1) is

$$
u=\frac{g_{1} f_{1}^{*}}{f_{1}^{2}}
$$

Since the equation (11) is homogeneous, we can assume

$g^{1}=e^{\tau_{1}}+e^{\tau_{2}}, \tau_{j}=\alpha_{j} t+\beta_{j} x+\eta_{j}^{0}, \beta_{j}^{2}=-\mathbf{i} \alpha_{j}, j=1,2$,

is a solution of (11), where $\alpha_{j}$ and $\eta_{j}^{0}$ are complex-valued constants. Substitute $g^{1}$ into (15), by Mathematica4.0 we can get

$f^{2}=e^{\tau_{1}+\tau_{1}^{*}+\vartheta_{13}}+e^{\tau_{1}+\tau_{2}^{*}+\vartheta_{14}}+e^{\tau_{2}+\tau_{1}^{*}+\vartheta_{23}}+e^{\tau_{2}+\tau_{2}^{*}+\vartheta_{24}}$,

where

$$
e^{\vartheta_{j, 2+l}}=\frac{\lambda}{2\left(\alpha_{j}^{2}-\alpha_{l}^{* 2}\right)}, j, l=1,2 .
$$

Substituting $g^{1}$ and $f^{2}$ into (12), we can get

$g^{3}=e^{\tau_{1}+\tau_{2}+\tau_{1}^{*}+\vartheta_{12}+\vartheta_{13}+\vartheta_{14}}+e^{\tau_{1}+\tau_{2}+\tau_{2}^{*}+\vartheta_{12}+\vartheta_{14}+\vartheta_{24}}$,

where

$e^{\vartheta_{12}}=-\frac{\mathbf{i} \lambda \alpha_{1} \alpha_{2}^{*}}{\alpha_{1}^{*}+\alpha_{2}}$

Substituting $g^{1}, g^{3}$ and $f^{2}$ into (16), we can get

$$
f^{4}=e^{\tau_{1}+\tau_{2}+\tau_{1}^{*}+\tau_{2}^{*}+\vartheta_{12}+\vartheta_{13}+\vartheta_{14}+\vartheta_{23}+\vartheta_{24}+\vartheta_{34}}
$$

where

$e^{\vartheta_{34}}=-e^{\vartheta_{12}^{*}}$

Substituting $g^{1}, g^{3}, f^{2}$ and $f^{4}$ into into (13), then (13) can be written to the former homogeneous equation (11), namely $g_{x x}^{5}+$ $\mathbf{i} g_{x}^{5}=0$. Then $g^{5}=0$ is a solution of $g_{x x}^{5}+\mathbf{i} g_{x}^{5}=0$, by virtue of (17), then we can get $f^{6}=0$.

The above procedure can be used to (11) - (18) again. So we can get $g^{7}=f^{8}=\cdots=0$. Hence we can infer, if $\varepsilon=1,(9)$ and (10) can be truncated to

$f_{2}(t, x)=1+e^{\tau_{1}+\tau_{1}^{*}+\vartheta_{13}}+e^{\tau_{1}+\tau_{2}^{*}+\vartheta_{14}}+e^{\tau_{2}+\tau_{1}^{*}+\vartheta_{23}}+e^{\tau_{2}+\tau_{2}^{*}+\vartheta_{24}}$

$+e^{\tau_{1}+\tau_{2}+\tau_{1}^{*}+\tau_{2}^{*}+\vartheta_{12}+\vartheta_{13}+\vartheta_{14}+\vartheta_{23}+\vartheta_{24}+\vartheta_{34}}$

$g_{2}(t, x)=e^{\tau_{1}}+e^{\tau_{2}}$

$+e^{\tau_{1}+\tau_{2}+\tau_{1}^{*}+\vartheta_{12}+\vartheta_{13}+\vartheta_{14}}+e^{\tau_{1}+\tau_{2}+\tau_{2}^{*}+\vartheta_{12}+\vartheta_{14}+\vartheta_{24}}$

So, the double soliton solution of $(1)$ is

$$
u=\frac{g_{2} f_{2}^{*}}{f_{2}^{2}} .
$$

Generally, if the solution of (11) is

$g^{1}=e^{\tau_{1}}+e^{\tau_{2}}+\cdots+e^{\tau_{n}}$

and

$\tau_{j}=\alpha_{j} t+\beta_{j} x+\eta_{j}^{0}, \beta_{j}^{2}=-\mathbf{i} \alpha_{j}, j=1,2, \cdots n$,

where $\alpha_{j}, \eta_{j}^{0}, \quad j=1,2, \cdots n$, are complex constants. Then, the $n$-soliton solutions of nonlinear Schrödinger equation of (1) are

$$
u=\frac{g_{n} f_{n}^{*}}{f_{n}^{2}}
$$


where

$$
\begin{aligned}
& f_{n}(t, x)=\sum_{\mu=0,1} A_{1}(\mu) \exp \left[\sum_{j=1}^{2 n} \mu_{j} \tau_{j}+\sum_{1 \leq j<l}^{2 n} \mu_{j} \mu_{l} \vartheta_{j l}\right] \\
& g_{n}(t, x)=\sum_{\mu=0,1} A_{2}(\mu) \exp \left[\sum_{j=1}^{2 n} \mu_{j} \tau_{j}+\sum_{1 \leq j<l}^{2 n} \mu_{j} \mu_{l} \vartheta_{j l}\right] \\
& \tau_{n+j}=\tau_{j}^{*},(j=1,2, \cdots, n)
\end{aligned}
$$

$e^{\vartheta_{j(n+l)}}=\frac{\lambda}{2\left(\alpha_{j}^{2}-\alpha_{l}^{* 2}\right)},(j, l=1,2, \cdots, n)$

$e^{\vartheta_{j l}}=-\frac{\mathbf{i} \lambda \alpha_{j} \alpha_{l}^{*}}{\alpha_{j}^{*}+\alpha_{l}},(j<l=2,3, \cdots, n)$,

$$
e^{\vartheta_{(n+j)(n+l)}}=-e^{\vartheta_{j l}^{*}},(j<l=2,3, \cdots, n),
$$

$A_{1}(\mu)$ and $A_{2}(\mu)$ satisfy

$A_{1}(\mu)= \begin{cases}1, & \text { if } \sum_{j=1}^{n} \mu_{j}=\sum_{j=1}^{n} \mu_{n+j}, \\ 0, & \text { others }\end{cases}$

$A_{2}(\mu)= \begin{cases}1, & \text { if } \sum_{j=1}^{n} \mu_{j}=\sum_{j=1}^{n} \mu_{n+j}+1, \\ 0, & \text { others, }\end{cases}$

the notation $\sum_{\mu=0,1}$ denotes the summation over all possible combination of $\mu_{1}=0,1, \mu_{2}=0,1, \cdots, \mu_{n}=0,1$, and (30) - (33) are calculated by Mathematica 4.0.

\section{Acknowledgement}

The authors appreciate the referee for his helpful and valuable comments on this manuscript.

The authors were supported by the Science Foundation of Binzhou University under grant BZXYL1406 and BZXYFB20150903 and by the Science and Technology Foundation of Shandong Province J16LI52.

\section{References}

[1] Ablowitz M J, Clarkson P A. Solitons, Nonlinear Evolution Equations and Inverse Scattering. Cambridge: Cambridge University Press, 1999.

[2] R. Beals and R. R. Coifman, Scattering and inverse scattering for first order systems I, II, Comm. pure. APPl. Math. 38(1985), 29-42.

[3] V. B. Matveev and M. A. Salle, Darboux transformation and solitons, Springer, Berlin, 1991.

[4] C. -H. Gu, H. -S. Hu and Z. -X. Zhou, Darboux Transformations in Soliton Theory and its Geometric Applications, Shanghai Science Technology Pubisher, Shanghai, 1991.

[5] Hirota R. Exact solution of the KdV equation formultiple collisions of solitons. Phys rev lett, 1971, 27: 1192-1194.

[6] W. -M. Liang. Sotiary wave solutions for variant Boussinesq equations.Phys Lett. A, 1995, 199(3-4): 169-172.

[7] L. -Y. Shen. Soliton and Integrable system. Shanghai: Shanghai Scientific and Technological Education Publishing House, 1999. 